\title{
THE UPTAKE OF CATECHOL AMINES AT HIGH PERFUSION CONCENTRATIONS IN THE RAT ISOLATED HEART: A NOVEL CATECHOL AMINE UPTAKE PROCESS
}

\author{
BY \\ L. L. IVERSEN* \\ From the Departments of Pharmacology and Biochemistry (Addenbrookes Hospital Section), University \\ of Cambridge
}

(Received August 30, 1964)

In previous studies of the uptake of catechol amines in the rat isolated heart it was shown that adrenaline and noradrenaline were accumulated by a common mechanism (Iversen, 1963, 1965). In these experiments the uptake saturated at an external amine concentration of approximately $0.2 \mu \mathrm{g} / \mathrm{ml}$. noradrenaline or $0.5 \mu \mathrm{g} / \mathrm{ml}$. adrenaline. However, in subsequent experiments in which hearts were perfused with $( \pm)$-adrenaline at a concentration of $5.0 \mu \mathrm{g} / \mathrm{ml}$. an unexpectedly large uptake of adrenaline was observed. This fortuitous observation led to an examination of the uptake of adrenaline and noradrenaline during perfusions at higher concentrations of each amine than had previously been studied.

The results of these experiments led to the conclusion that a second type of uptake operates at high perfusion concentrations. The properties of this second process are sufficiently different from those described previously to justify a clear-cut distinction between the two processes.

\section{METHODS}

The methods used for studying catechol amine uptake in the rat isolated heart during perfusion with a medium containing added adrenaline or noradrenaline have been described in detail elsewhere (Iversen, $1963,1965)$. In the present experiments the uptakes of adrenaline and noradrenaline were so large that it was usually possible to measure the uptake accurately by fluorimetric analysis alone. The catechol amine content of purified heart extracts was assayed by a differential fluorimetric procedure (Iversen, 1963).

In a few experiments in which $( \pm)$-[ $\left.{ }^{3} \mathrm{H}\right]$-noradrenaline was used, the concentration of tritium added to the perfusion medium was $45 \mathrm{~m} \mu \mathrm{c} / \mathrm{ml}$. - ten-times that used in previous experiments. This was necessary because of the relatively small proportion of the perfused material removed during passage through the tissue. In these experiments the full ion-exchange chromatography method (Iversen, 1963) was used to isolate [ $\left.{ }^{3} \mathrm{H}\right]$-noradrenaline from the heart extracts in order to avoid including tritiated metabolites in the $\left[{ }^{3} \mathrm{H}\right]$-noradrenaline estimations.

\footnotetext{
* Present address: Laboratory of Clinical Science, National Institute of Mental Health, National Inst itutes of Health, Bethesda, Maryland, U.S.A.
} 
It was not possible to use the final 2-min wash-out procedure employed to remove extracellular catechol amines in previous exferiments, because of the rapid wash-out of accumulated adrenaline or noradrenaline in the present experiments. Uptake measurements were made on hearts removed from the perfusion apparatus immediately following the catechol amine perfusion, unless otherwise stated. The catechol amine content of the tissue was corrected for the presence of catechol amines in the extracellular water space. The extracellular space in well-blotted tissue was determined by estimating the $\left.{ }^{4} \mathrm{C}\right]$ content of hearts after a 10-min perfusion with a medium containing a krown concentration of [' $\mathrm{C}$ ]-sorbitol (obtained from the Radiochemical Centre, Amersham, Bucks). These experiments gave a value of $325 \pm 22 \mu 1 . / \mathrm{g}$ for the extracellular space (mean and standard error of six perfusions). This is identical with the figure obtained by Randle (personal communication) using a similar perfusion technique. After perfusion with $\left[{ }^{14} \mathrm{C}\right]$-sorbitol for only $1 \mathrm{~min}$, more than $94 \%$ of the extracellular space was found to have equilibrated; Morgan, Henderson, Regen \& Park (1961) have described a similarly rapid equilibration of the extracellular space in this preparation.

In experiments in which noradrenaline uptake was measured by fluorimetric assay, the noradreraline content of the heart was also corrected for the presence of endogenous noradrenaline, using the average value of $0.96 \mu \mathrm{g}$ of noradrenaline per $\mathrm{g}$ of heart previously determined (Iversen, 1963). The uptake results therefore refer to the net increases in the intracellular noradrenaline or adrenaline content of the tissue. All values are the mean results for groups of from four to six hearts.

\section{RESULTS}

\section{General characteristics of catechol amine uptake}

A previous kinetic analysis of the uptake of $( \pm)$-adrenaline in the rat isolated heart showed that in a range of perfusion concentrations from 0.02 to $0.50 \mu \mathrm{g} / \mathrm{ml}$. the uptake of adrenaline was by a saturable process which reached a maximum rate of $0.13 \mu \mathrm{g} / \mathrm{min} / \mathrm{g}$ of heart (Iversen, 1965). From the application of Michaelis-Menten kinetics to these results the maximum rate of adrenaline uptake at infinitely high perfusion concentrations $\left(V_{\text {max }}\right)$ was estimated to be $0.19 \mu \mathrm{g} / \mathrm{min} / \mathrm{g}$ of heart. However, as shown in Fig. 1, the rates of uptake at perfusion concentrations greater than $0.5 \mu \mathrm{g} / \mathrm{ml}$. adrenaline were higher than this maximum rate. The uptake of (上)-adrenaline was therefore studied in more detail in a

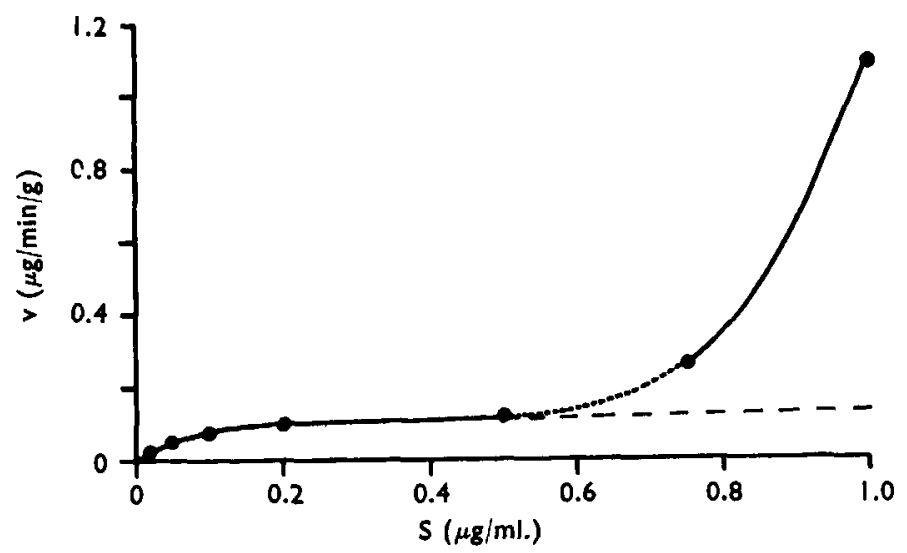

Fig. 1. Initial rates of adrenaline uptake $(v, \mu \mathrm{g} / \mathrm{min} / \mathrm{g}$, ordinate) by the rat isolated heart at various perfusion concentrations of $( \pm)$-adrenaline $(\mathrm{S}, \mu \mathrm{g} / \mathrm{ml}$., abscissa). The maximum rate of adrenaline uptake predicted from a previous study is indicated by the dashed line. The values for rates of adrenaline uptake at perfusion concentrations not exceeding $0.5 \mu \mathrm{g} / \mathrm{ml}$. adrenaline are also taken from this study (Iversen, 1965). 


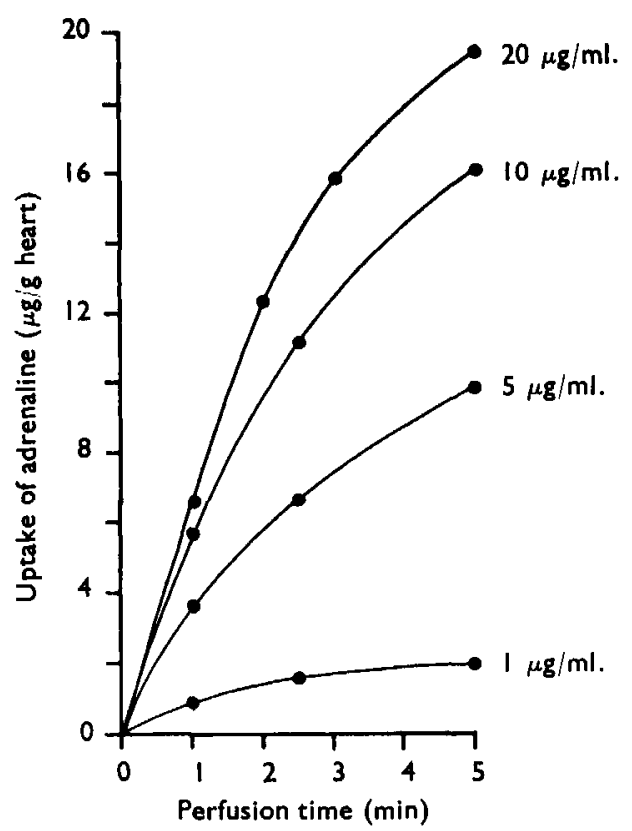

Fig. 2. Uptake curves for adrenaline (ordinate, $\mu \mathrm{g} / \mathrm{g}$ of heart) at high perfusion concentrations of (1)-adrenaline (values on right) in the rat isolated heart. Abscissa, perfusion time (min). Perfusion concentrations ranged from 1 to $20 \mu \mathrm{g} / \mathrm{ml}$. adrenaline. Each point is the mean value for a group of six hearts.

series of experiments in which rat hearts were perfused with various concentrations of $( \pm)$-adrenaline in the range from 0.75 to $40.00 \mu \mathrm{g} / \mathrm{ml}$. Some of these results are illustrated in Fig. 2. There was a rapid accumulation, amounting in several cases to more than $10 \mu \mathrm{g}$ of adrenaline per $\mathrm{g}$ of tissue, or more than ten-times the total endogenous catechol amine content of the heart.

Except at the highest perfusion concentrations, a concentration ratio of accumulated adrenaline per $\mathrm{g}$ of tissue over external adrenaline per $\mathrm{ml}$. of medium of more than 1.0 was attained after only very short periods of perfusion. The uptake of adrenaline at a perfusion concentration of $5 \mu \mathrm{g} / \mathrm{ml}$. was studied in more detail in perfusions up to $20 \mathrm{~min}$ in duration (Fig. 3,a). The uptake of adrenaline rapidly approached an asymptotic value of approximately $14 \mu \mathrm{g} / \mathrm{g}$ adrenaline, indicating that a concentration ratio of almost $3: 1$ was attained. From the curve shown in Fig. 3,a it was possible to estimate the rate of adrenaline uptake at various times during the perfusion, and these values were plotted against time on semi-log paper (Iversen, 1963). The result, shown in Fig. 3,b, was a straight line, indicating that the rate of adrenaline uptake declined exponentially with time. Uptake curves for adrenaline at other perfusion concentrations (for example, Fig. 2) were also analysed in this way and in all instances a single straight line was the result. The uptake curves for adrenaline could thus be described by a simple exponential equation of the form

$$
C=C_{\infty}\left(1-e^{-a t}\right)
$$

where $C$ is the tissue content at time $\mathrm{t}, \alpha$ is the rate constant of the uptake process, and $C_{\infty}$ is the tissue content at time $t=\infty$. This equation was used to calculate the $C_{\infty}$ values 
(a)

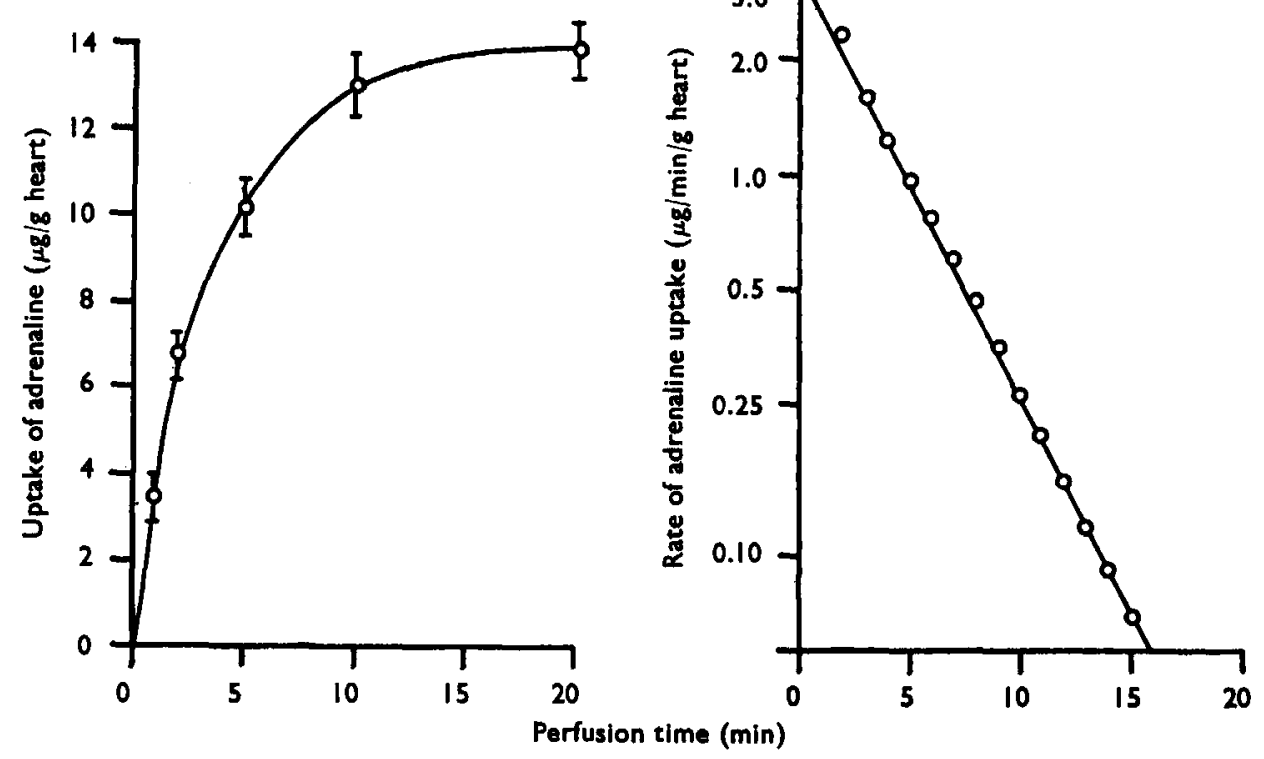

Fig. 3. (a) The uptake of adrenaline (ordinate, $\mu \mathrm{g} / \mathrm{g}$ of heart) at a perfusion concentration of $5 \mu \mathrm{g} / \mathrm{ml}$. of $( \pm$ )-adrenaline in the rat isolated heart. Values are the means and standard errors for groups of six hearts. (b) Rates of adrenaline uptake (ordinate, $\mu \mathrm{g} / \mathrm{min} / \mathrm{g}$ of heart) (derived from a) at vatious times during perfusion of the rat isolated heart with $( \pm)$-adrenaline at a concentration of $5 \mu \mathrm{g} / \mathrm{ml}$. Abscissa, perfusion time (min).

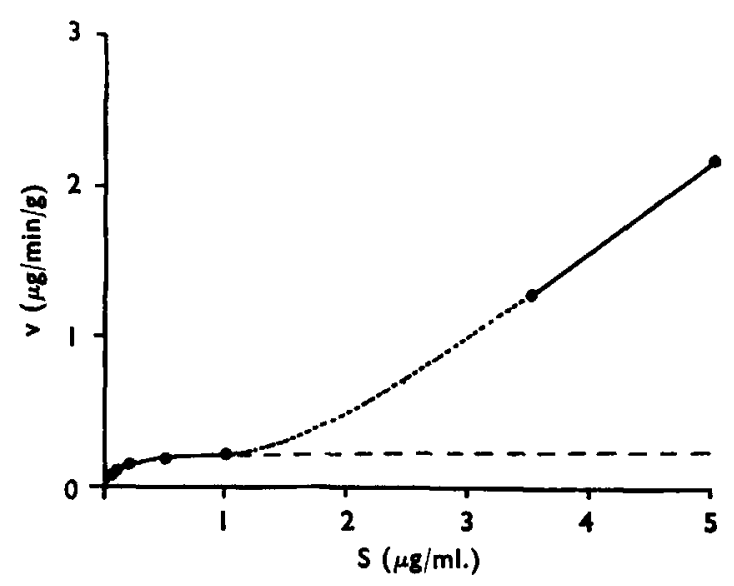

Fig. 4. Initial rates of noradrenaline uptake $(v, \mu \mathrm{g} / \mathrm{min} / \mathrm{g}$, ordinate) by the rat isolated heart at various perfusion concentrations of $( \pm)$-noradrenaline $(\mathrm{S}, \mu \mathrm{g} / \mathrm{ml}$., abscissa). The values for perfusion concentrations not exceeding $1 \mu \mathrm{g} / \mathrm{ml}$. are taken from Iversen (1963) and the maximum rate of noradrenaline uptake predicted from this previous study is indicated by the dashed line. 
for adrenaline at other perfusion concentrations from the incomplete uptake curves available (Fig. 2). For concentrations of $1,5,10,20$ and $40 \mu \mathrm{g} / \mathrm{ml}$., the $C_{\infty}$ values were 2.2, 14.6, 20.1, 23.7 and $28.8 \mu \mathrm{g} / \mathrm{g}$ respectively.

As shown in Fig. 4, noradrenaline was also accumulated very rapidly by the rat heart at high perfusion concentrations. In similar experiments the uptake of $( \pm$ )-noradrenaline was studied at perfusion concentrations in the range of 3.5 to $40.0 \mu \mathrm{g} / \mathrm{ml}$. It was found that noradrenaline was accumulated in a manner qualitatively similar to adrenaline under these perfusion conditions.

\section{Kinetic analysis of initial rates of adrenaline and noradrenaline uptake}

Initial rates of uptake were determined from the uptake curves for adrenaline and noradrenaline at various perfusion concentrations by extrapolating the rate analysis plots (for example, Fig. $3, b$ ) to zero time. Even curves which extended over only very short time intervals (for example, Fig. 2) were adequate for obtaining estimates of the initial rates of uptake.

The initial rates of uptake were plotted against perfusion concentrations (Fig. 5) and these values were also plotted in a form of the Michaelis-Menten equation as S/v against S (Fig. 6). The accuracy with which a straight line fitted the results when plotted in this manner showed that this type of kinetic equation was adequate to describe the uptake. Kinetic constants for adrenaline and noradrenaline uptake were determined from Fig. 6 and are summarized

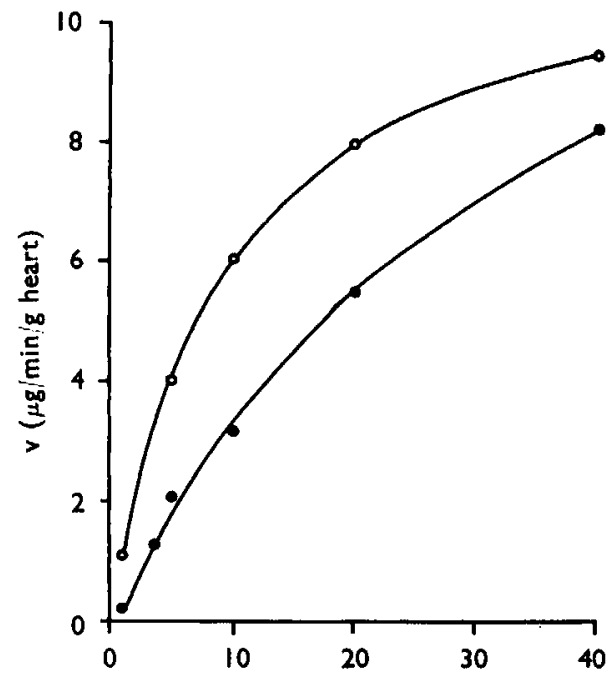

Fig. 5. The initial rates of adrenaline $(O)$ and noradrenaline (๑) uptake $(v, \mu \mathrm{g} / \mathrm{min} / \mathrm{g}$ heart, ordinate) in the rat isolated heart perfused with various concentrations of $( \pm)$-adrenaline or ( \pm )-noradrenaline in the range from 1 to $40 \mu \mathrm{g} / \mathrm{ml}$. (S, $\mu \mathrm{g} / \mathrm{ml}$., abscissa).

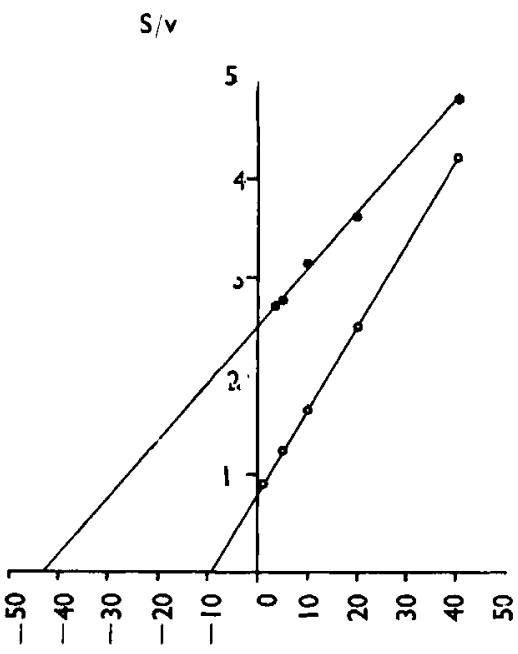

Fig. 6. Michaelis-Menten analysis of the results in Fig. 5 plotted as $S / v$ (ordinate) against $S$ (abscissa). $\mathbf{S}=$ =perfusion concentration of adrenaline $(O)$ or noradrenaline $(\bullet) ; \quad v=$ initial rate of adrenaline or noradrenaline uptake by the isolated heart. Kinetic constauts were determined as follows: intercept on baseline $=-K_{m} ; \quad$ slope $=1 / V_{\max } ;$ and intercept on vertical axis $=K_{m} / V_{m a x^{*}}$ 
TABLE 1

\section{KINETIC CONSTANTS FOR SECOND UPTAKE PROCESS}

$K_{m}$ and $V_{\max }$ were determined from Fig. 6 after lines of best fit had been drawn by the method of least squares analysis, and standard errors of slope were determined and used to calculate the standard errors (Pearl, 1940). The figures in brackets are the kinetic constants for noradrenaline and adrenaline uptake at lower perfusion concentrations (Iversen, 1963, 1965). Standard errors for $V_{\max }$ are given

$\begin{array}{ccc}\text { Compound } & \begin{array}{c}\text { Affinity constant for } \\ \text { second uptake process } \\ K_{m}\left(M \times 10^{-6}\right)\end{array} & \begin{array}{c}V_{\max } \\ (\mu \mathrm{g} / \mathrm{min} / \mathrm{g})\end{array} \\ ( \pm) \text {-Noradrenaline } & 252 \cdot 0 & 17 \cdot 0 \pm 0.36 \\ & (0.66) & (0.24) \\ ( \pm) \text {-Adrenaline } & 51.6 & 11 \cdot 8 \pm 0.25 \\ & (1 \cdot 40) & (0 \cdot 19)\end{array}$

in Table 1. Table 1 also includes the kinetic constants for adrenaline and noradrenaline uptake previously determined at lower perfusion concentrations. In contrast to the uptake process at lower perfusion concentrations previously described, in the present experiments $( \pm)$-adrenaline had a considerably higher affinity than ( \pm -noradrenaline. The affinities of both adrenaline and noradrenaline in the second uptake process were very much lower than their affinities in the process described earlier (Iversen, 1963, 1965). However, the maximum rates of catechol amine uptake by the second process were much higher than those previously found.

\section{Uptake of catechol amines followed by perfusion with catechol amine-free solutions}

After a perfusion with $( \pm)$-adrenaline at a concentration of $5 \mu \mathrm{g} / \mathrm{ml}$. for $10 \mathrm{~min}$, perfusion was continued for various periods with an adrenaline-free medium. These experiments

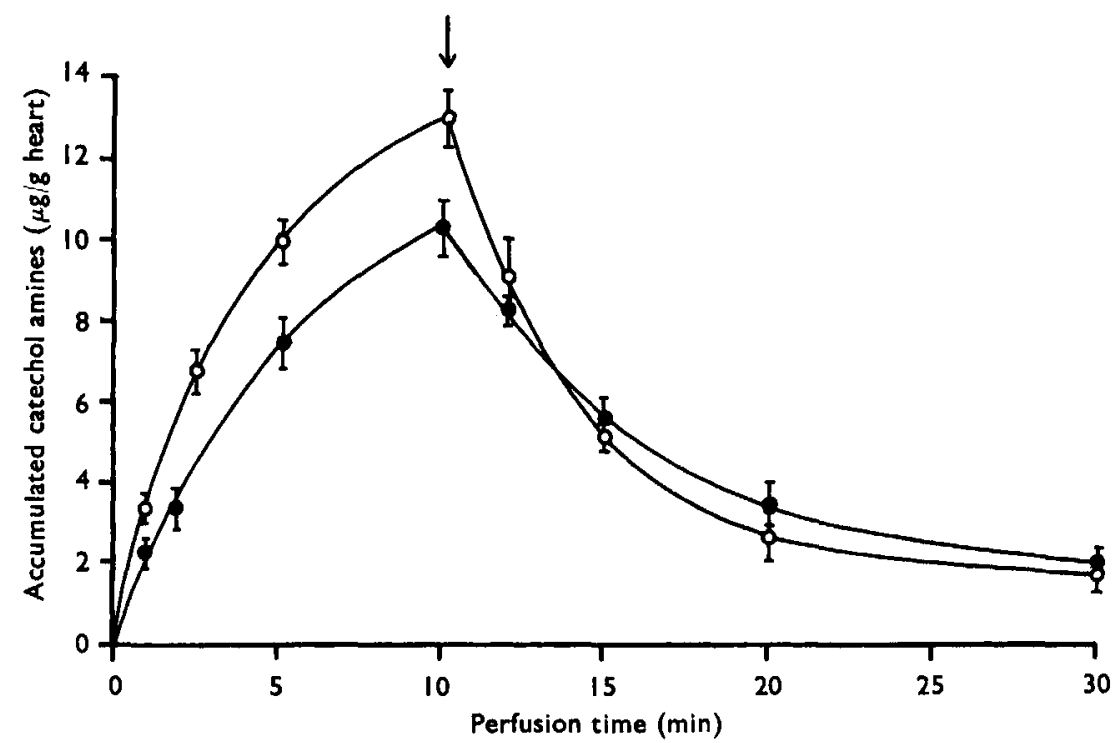

Fig. 7. The disappearance of catechol amines from the rat heart when perfusion for $10 \mathrm{~min}$ (period 0 to $10 \mathrm{~min}$ ) with a medium containing ( $t$ )-adrenaline or ( $t$ )-noradrenaline at a concentration of $5 \mu \mathrm{g} / \mathrm{ml}$. was followed by various periods of perfusion with a catechol amine-free medium (starting at arrow). Each point is the mean value for a group of six hearts; vertical bars indicate standard errors of the means. Empty circles, adrenaline; filled circles, noradrenaline. 
(Fig. 7) showed that the adrenaline content of the tissue decreased rapidly until after 20 min a residue of approximately $2 \mu \mathrm{g} / \mathrm{g}$ adrenaline remained in the heart in a form resistant to further wash-out. Similar results were obtained in analogous experiments with noradrenaline.

The curves illustrated in Fig. 7 were also analysed in a manner similar to that described for adrenaline and noradrenaline uptake. When rates of adrenaline and noradrenaline disappearance were plotted against time on semi-log paper, they yielded straight lines approximately parallel to those obtained for uptake (Fig. 8).

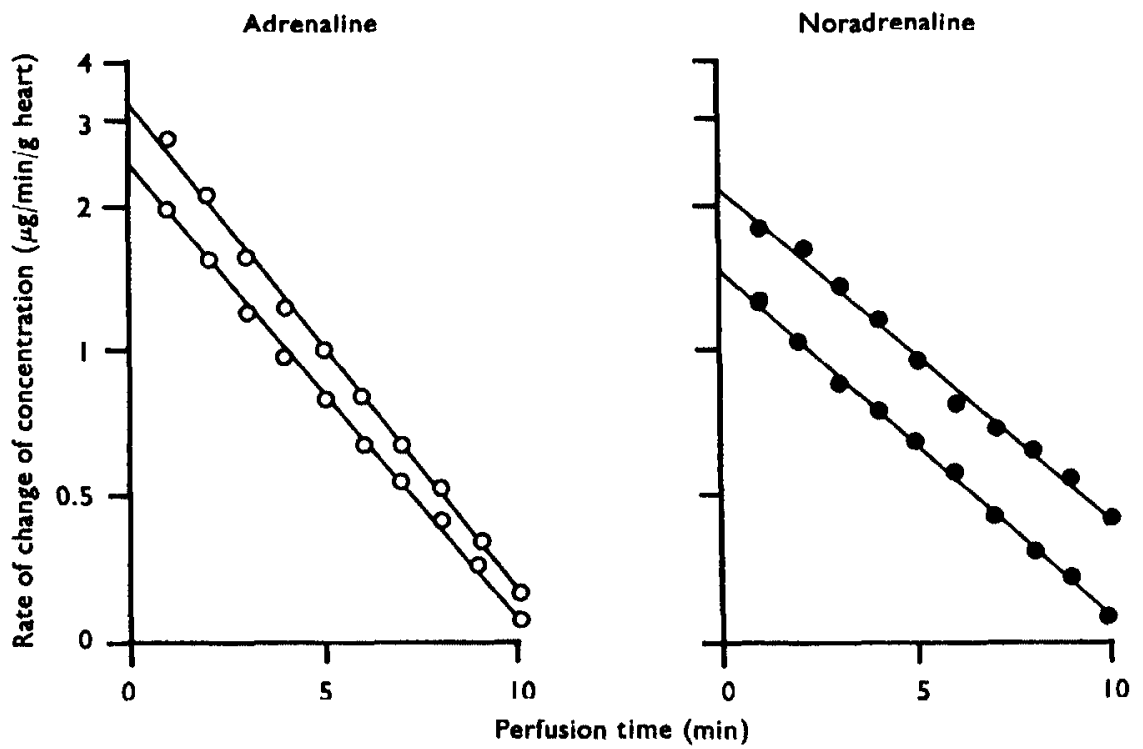

Fig. 8. Rate analysis of the uptake (upper line on each graph) and disappearance (lower line on each graph) curves illustrated in Fig. 7. The rate of catechol amine uptake or disappearance is plotted as the ordinate, irrespective of sign, against time. The uptake and disappearance plots do not exactly superimpose because of the residual amount of accumulated catechol amine which remained in the tissue after prolonged periods of perfusion with a catechol amine-free medium.

\section{Stereochemical specificity}

Groups of hearts were perfused for 2 min with (+)-noradrenaline or (-)-noradrenaline at a concentration of $5 \mu \mathrm{g} / \mathrm{ml}$. The uptakes of the two enantiomers were not significantly different (Table 2). A similar experiment with ( - )-adrenaline and ( \pm )-adrenaline indicated a slightly higher uptake after perfusion with the laevorotatory enantiomer but this difference was not statistically significant (Table 2).

\section{Inhibition by drugs of noradrenaline uptake and removal}

In these experiments groups of hearts were perfused with a medium containing $5 \mu \mathrm{g} / \mathrm{ml}$. of $( \pm)$-[ $\left.{ }^{3} \mathrm{H}\right]$-noradrenaline for $2 \mathrm{~min}$. Drugs were added to the perfusion medium so that the heart was exposed to the drug and to $\left[{ }^{3} \mathrm{H}\right]$-noradrenaline simultaneously. The results were expressed as the percentage inhibition of noradrenaline uptake in drug-treated groups 
TABLE 2

\section{UPTAKE OF OPTICAL ISOMERS OF ADRENALINE AND NORADRENALINE BY SECOND} UPTAKE PROCESS

Perfusions were all at a concentration of $5 \mu \mathrm{g} / \mathrm{ml}$. Tissue contents are means and standard errors of determinations after perfusion, and are corrected for extracellular space. Noradrenaline uptake figures are corrected for the presence of $0.96 \mu \mathrm{g} / \mathrm{g}$ endogenous noradrenaline in the heart. Number of animals in each group is indicated by the figure in brackets. $P$ values for comparisons of ( - )-adrenaline and $( \pm)$-adrenaline, and of $(-)$-noradrenaline and $(+)$-noradrenaline, were greater than 0.3

$\begin{array}{ccc}\text { Compounds } & \begin{array}{c}\text { Perfusion time } \\ (\mathrm{min})\end{array} & \begin{array}{c}\text { Tissue content } \\ (\mu \mathrm{g} / \mathrm{g} \text { heart) }\end{array} \\ \text { 士)-Adrenaline } & 2 \cdot 5 & 6.65 \pm 0.41(6) \\ \text {-)-Adrenaline } & 2 \cdot 5 & 7.28 \pm 0.34(6) \\ \text {-)-Noradrenaline } & 2 \cdot 0 & 3.26 \pm 0.14(6) \\ \pm)- \text { Noradrenaline } & 2.0 & 3.35 \pm 0.10(12) \\ + \text { )-Noradrenaline } & 2.0 & 3.55 \pm 0.23(6)\end{array}$

compared with that in drug-free controls. The uptake of $\left[{ }^{3} \mathbf{H}\right]$-noradrenaline was inhibited by cocaine, phenoxybenzamine, desipramine, normetanephrine and metanephrine, but not by metaraminol. These experiments are summarized in Table 3, which includes results obtained when these drugs acted as inhibitors of the noradrenaline uptake at lower perfusion concentrations.

The removal of noradrenaline during perfusion with a noradrenaline-free medium could also be inhibited. A group of hearts was first perfused with ( \pm )-noradrenaline at a concentration of $5 \mu \mathrm{g} / \mathrm{ml}$. for $10 \mathrm{~min}$, perfusion was then continued with a noradrenalinefree medium to which $( \pm)$-metanephrine had been added to a final concentration of $10^{-4} \mathrm{M}$. Under these conditions the removal of noradrenaline was completely inhibited. In similar experiments the presence of (-)-metaraminol at a concentration of $10^{-4} \mathrm{M}$ had no significant effect. These results are illustrated in Fig. 9.

\section{Competition between noradrenaline and adrenaline for uptake}

In these experiments hearts were perfused with (土)-[ $\left.{ }^{3} \mathrm{H}\right]$-noradrenaline at a concentration of $5 \mu \mathrm{g} / \mathrm{ml}$. for $2 \mathrm{~min}$. The uptake of $\left[{ }^{3} \mathrm{H}\right]$-noradrenaline in control experiments

TABLE 3

\section{INHIBITION OF NORADRENALINE UFTAKE BY DRUGS}

The uptake of $\left[{ }^{8} \mathrm{H}\right]$-noradrenaline at a concentration of $5 \mu \mathrm{g} / \mathrm{ml}$. was measured after a 2-min perfusion in the presence of inhibitors. The uptake of $\left[{ }^{2} \mathrm{H}\right]$-noradrenaline in a group of twenty control hearts was $3.21 \pm 0.23 \mu \mathrm{g} / \mathrm{g}$ (mean and standard error). Each drug concentration was tested on a group of four hearts. The values for inhibition of $\left[{ }^{8} \mathrm{H}\right]$-noradrenaline at lower perfusion concentrations were determined by the method previously described (Iversen, 1964)

\section{Drug \\ Drug concentration}

Cocaine

Cocaine

Desipramine

Desipramine

Phenoxybenzamine

(-)-Metaraminol

(-)-Metaraminol

( $)$-Normetanephrine

(士)-Normetanephrine

(I)-Metanephrine

(士)-Metanephrine
(M)

$1 \times 10^{-6}$

$2 \times 10^{-4}$

$1 \times 10^{-7}$

$2 \times 10^{-4}$

$1 \times 10^{-5}$

$2 \times 10^{-7}$

$2 \times 10^{-4}$

$2 \times 10^{-6}$

$2 \times 10^{-8}$

$2 \times 10^{-6}$

$2 \times 10^{-5}$
Inhibition $(\%)$ of $\left[{ }^{a} \mathrm{H}\right]$-noradrenaline uptake for concentration

\begin{tabular}{cr}
\hline $5 \mu \mathrm{g} / \mathrm{ml}$. & $10 \mathrm{ng} / \mathrm{ml}$. \\
\hline 34 & 71 \\
$\overline{79}$ & 100 \\
55 & 81 \\
- & 100 \\
3 & 89 \\
23 & 69 \\
85 & 100 \\
40 & 8 \\
92 & 31 \\
& -31
\end{tabular}




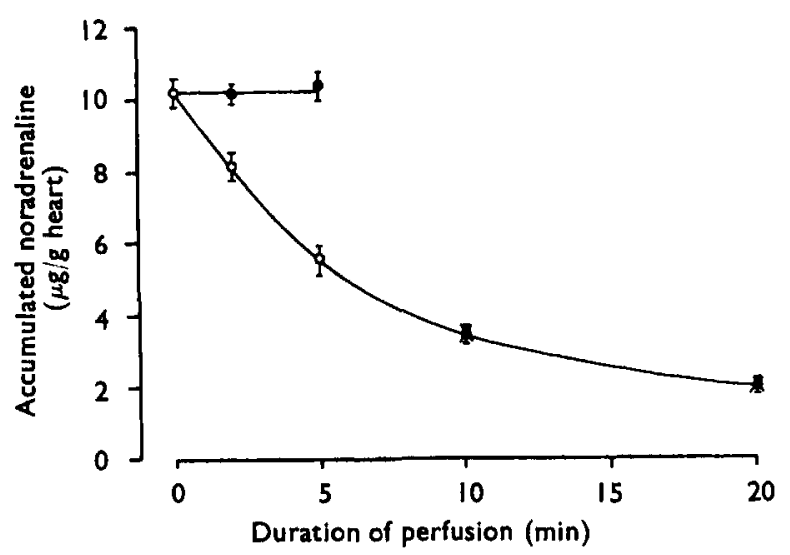

Fig. 9. The effect of drugs on the disappearance of accumulated noradrenaline from the rat isolated heart during perfusion with a noradrenaline-free medium. Perfusion for a 10-min period with ( $t$ )-noradrenaline at a concentration of $5 \mu \mathrm{g} / \mathrm{ml}$. was followed by various periods of perfusion with a catechol amine-free medium (starting at zero time). ( \pm )-Metanephrine or ( - )-metaraminol was added to this medium to final concentrations of $10^{-4} \mathrm{M}$. Each point is the mean value for a group of six hearts; vertical lices indicate standard errors of the means. Empty circles, control values, taken from Fig. 7; filled circles, (土)-metanephrine; crosses, (-)-metaraminol.

(Table 3) agreed closely with the net uptake of noradrenaline under these perfusion conditions, as previously measured by fluorimetric analysis (Table 2). The uptake of $\left[{ }^{3} \mathrm{H}\right]$-noradrenaline was also measured in groups of hearts perfused with $\left[{ }^{3} \mathrm{H}\right]$-noradrenaline together with various amounts of nonradioactive ( \pm )-adrenaline. In the presence of adrenaline the uptake of $\left[{ }^{3} \mathrm{H}\right]$-noradrenaline was inhibited. The obvious explanation of these results is that adrenaline and noradrenaline compete for a common mechanism of uptake. If this is true then the inhibition of $\left[{ }^{3} \mathrm{H}\right]$-noradrenaline uptake by adrenaline should be related to the affinity properties of adrenaline in the uptake process. Since the concentration of noradrenaline used produced relatively little saturation of the uptake mechanism, the concentration of adrenaline producing a $50 \%$ inhibition of noradrenaline uptake should be approximately equal to the concentration found to produce a half saturation of the uptake $\left(K_{m}\right)$ in the previous experiments illustrated in Figs. 5 and 6. In Fig. 10, the percentage inhibition of $\left[{ }^{3} \mathrm{H}\right]$-noradrenaline uptake is plotted against the log concentration of added adrenaline. From this figure the $50 \%$ inhibitory concentration is seen to be $9.1 \mu \mathrm{g} / \mathrm{ml}$. $\left(5.0 \times 10^{-5} \mathrm{M}\right)$; this corresponds closely to the previously determined value of $K_{m}$ for adrenaline (Table 1).

The relation of the uptake of catechol amines at high perfusion concentrations to that previously described at lower perfusion concentrations

The uptake of $\left[{ }^{3} \mathrm{H}\right]$-adrenaline under conditions which favoured the operation of each type of uptake process was studied in the experiment illustrated in Fig. 11. The uptake of a tracer concentration of $( \pm)-\left[{ }^{3} \mathrm{H}\right]$-adrenaline $(2.5 \mathrm{ng} / \mathrm{ml}$. adrenaline $)$ was measured by radioactivity analysis in the presence of various amounts of nonradioactive ( \pm )-noradrenaline added simultaneously to the perfusion medium. As the concentration of added noradrenaline approached $0.5 \mu \mathrm{g} / \mathrm{ml}$. there was a progressive decline in the uptake of 


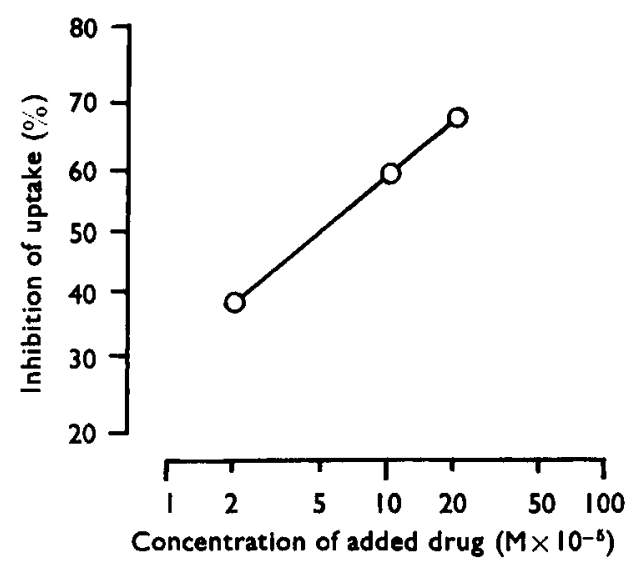

Fig. 10. The inhibition of $(\exists)$-[ $\left[{ }^{3} \mathrm{H}\right]$-roradrenaline uptake at a perfusion concentration of $5 \mu \mathrm{g} / \mathrm{ml}$. noradrenaline by the addition of various amounts of nonradioactive $( \pm$ )-adrenaline to the perfusion medium (abscissa, $\mathrm{M} \times 10^{-5}, \log$ scale). $\left[{ }^{3} \mathrm{H}\right]-$ Noradrenaline uptake was measured after a 2 -min perfusion in each experiment. Results are expressed as percentage inhibition of $\left[{ }^{8} \mathrm{H}\right]$-noradrenaline uptake (ordinate, probability scale) in adrenaline-treated groups compared with adrenaline-free controls; each point is the mean value for a group of from four to six hearts.

$\left[{ }^{3} \mathrm{H}\right]$-adrenaline. This was expected because of the competition between noradrenaline and adrenaline for the first uptake process, which led to a progressive saturation of this system by increasing concentrations of noradrenaline. However, as the concentration of added noradrenaline exceeded $0.5 \mu \mathrm{g} / \mathrm{ml}$., and the second uptake system came into operation, the direction of the curve was reversed and the fraction of $\left[{ }^{3} \mathrm{H}\right]$-adrenaline uptake increased with increasing noradrenaline concentrations. At concentrations above $5 \mu \mathrm{g} / \mathrm{ml}$. noradrenaline a second phase of inhibition of $\left[{ }^{3} \mathrm{H}\right]$-adrenaline uptake was seen, corresponding to the competitive depression of uptake described in the previous paragraph.

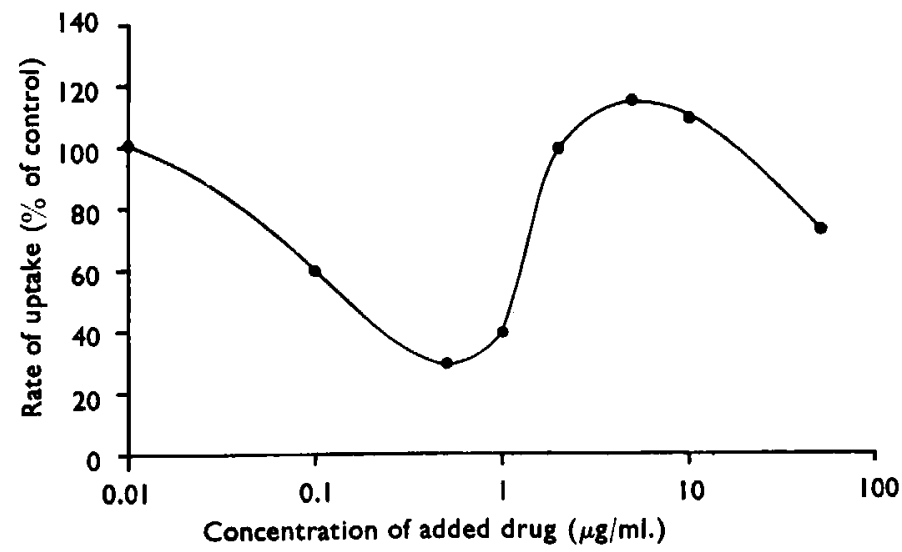

Fig. 11. The uptake of $\left[{ }^{2} \mathrm{H}\right]$-adrenalire in the rat isolated heart perfused with a medium containing a tracer concentration of $\left(t^{-}\right)-\left[^{3} \mathrm{H}\right]$-adrenaline $(2.5 \mathrm{ng} / \mathrm{ml}$.), to which various amounts of nonradioactive $( \pm)$-noradreraline vere added (abscissa, $\log s c a l e, \mu \mathrm{g} / \mathrm{ml}$.). The uptake of $\left[{ }^{3} \mathrm{H}\right]$-adrenaline (ordinate) is expressed as the percentage of $\left[{ }^{3} \mathrm{H}\right]$-adrenaline uptake in a control experiment in which no noradrenaline was added; each point is the mean value for a group of four to six hearts. 
The uptake of noradrenaline in hearts from " immunosympathectomized" rats

Hearts from rats treated with the antiserum against the sympathetic nerve growth factor, as described by Levi-Montalcini \& Angeletti (1962), were studied. This procedure has been shown to cause a striking atrophy of sympathetic ganglion cells and postganglionic neurones and an extensive reduction of the noradrenaline content of the heart and spleen. The animals were of the Hooded Lister strain and weighed around $100 \mathrm{~g}$. The efficiency of the antibody treatment was assessed by measurements of the noradrenaline content of the spleen. In all six animals the noradrenaline content was less than $0.05 \mu \mathrm{g} / \mathrm{g}$.

Hearts from two of the animals were used to test the effects of the immunosympathectomy on the uptake of noradrenaline at low perfusion concentrations. These hearts were perfused with $( \pm)-\left[{ }^{3} \mathrm{H}\right]$-noradrenaline at a concentration of $1 \mathrm{ng} / \mathrm{ml} .(9 \mathrm{~m} \mu \mathrm{c} / \mathrm{ml}$.) for $10 \mathrm{~min}$. After a final 2-min wash-out perfusion with noradrenaline-free medium to remove extracellular $\left[{ }^{3} \mathrm{H}\right]$-noradrenaline, the $\left[{ }^{3} \mathrm{H}\right]$-noradrenaline content of the tissue was estimated. The uptake of $\left[{ }^{3} \mathrm{H}\right]$-noradrenaline in the hearts from the treated animals was approximately $6 \%$ of the uptake in three control hearts perfused under the same conditions $(0.85 \mathrm{ng} / \mathrm{g}$ compared with $14.10 \mathrm{ng} / \mathrm{g}$ ). The uptake of $\left[{ }^{3} \mathrm{H}\right]$-noradrenaline was also estimated in these experiments by measuring the amount of $\left[{ }^{3} \mathrm{H}\right]$-noradrenaline removed from the perfusate during its passage through the heart. In the treated hearts the rates of noradrenaline uptake were too small to give a measurable difference in concentration between the inflow fluid and the effluent. In control animals, some $30 \%$ of the noradrenaline was removed. Since only tracer amounts of $\left[{ }^{3} \mathrm{H}\right]$-noradrenaline were used in these experiments, it was possible to measure the endogenous noradrenaline content of the hearts from the treated animals by a fluorimetric analysis of the purified extracts, and no noradrenaline $(<0.05 \mu \mathrm{g} / \mathrm{g} \mathrm{com-}$ pared with $0.90 \mu \mathrm{g} / \mathrm{g}$ for the three controls) could be detected in the treated hearts analysed in this way.

The hearts from the remaining four animals were used to test the effects of the immunosympathectomy on the uptake of noradrenaline at high perfusion concentrations. These hearts were perfused for 2 min with a medium containing ( \pm )-noradrenaline at a concentration of $5 \mu \mathrm{g} / \mathrm{ml}$,, and the uptake of noradrenaline was then measured by a fluorimetric analysis of the purified tissue extracts. The uptake of noradrenaline in the hearts from the treated animals amounted to approximately $20 \%$ of that in control hearts under the same conditions $(0.70 \mu \mathrm{g} / \mathrm{g}$, range 0 to $1.2 \mu \mathrm{g} / \mathrm{g}$, compared with $3.35 \pm 0.10$, mean and standard error of twelve controls).

\section{DISCUSSION}

The uptake of catechol amines in the rat isolated heart at high perfusion concentrations was mediated by a mechanism qualitatively and quantitatively different from that responsible for the uptake of catechol amines at lower perfusion concentrations (Iversen, 1963, 1965). To facilitate the discussion of these results it is proposed that the process previously described, operative at low concentrations, be termed Uptake ${ }_{1}$, and that described in the present experiments, which operates at high concentrations, be termed Uptake ${ }_{2}$.

Both processes saturate with increasing external amine concentrations and can be described by Michaelis-Menten kinetics. The affinity of $( \pm)$-noradrenaline for Uptake is 374-times less than its affinity for Uptake ${ }_{1}$, and the affinity of $( \pm)$-adrenaline for Uptake is 37-times less than for Uptake . Uptake $_{2}$ favoured the accumulation of adrenaline 


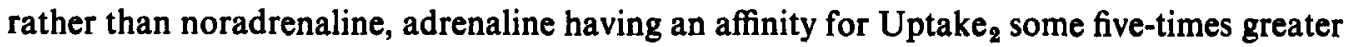
than that of noradrenaline. On the other hand, Uptake $_{1}$ favoured the accumulation of noradrenaline which had more than twice the affinity of adrenaline. The maximum rates of noradrenaline and adrenaline accumulation by Uptake $_{2}$ are some sixty- to seventy-times greater than the maximum rates of accumulation by Uptake ${ }_{1}$. These differences in the kinetic properties of the two systems are summarized in Table 1. Uptake ${ }_{2}$ did not discriminate between the optical isomers of adrenaline and noradrenaline, whereas Uptake favoured the accumulation of the laevorotatory stereoisomers. Furthermore, a large proportion of the catechol amines accumulated by Uptake ${ }_{2}$ was removed from the heart during perfusion with a catechol amine-free medium, in contrast to the remarkable resistance of catechol amines accumulated by Uptake , $_{1}$ to such treatment.

Cocaine and desipramine produced some inhibition of both uptake processes (Table 3 ). Much higher concentrations of these drugs, however, were needed to inhibit Uptake than were required to inhibit Uptake ${ }_{1}$. This may be partly due to the presence of much larger amounts of noradrenaline in the test procedure used to assess drug inhibition of Uptake $_{2}$, but may also reflect differences in the drug sensitivities of the two processes. Despite the much higher concentrations of noradrenaline used to test drug inhibition of Uptake $_{2}$, phenoxybenzamine at a concentration of $10^{-5} \mathrm{M}$ produced an appreciable inhibition of both uptake processes. (-)-Metaraminol, which was previously found to be a

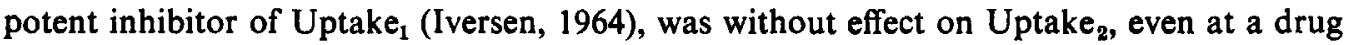
concentration 500-times greater than that required to produce an almost complete inhibition of Uptake . On the other hand, (土)-metanephrine was more effective as an inhibitor of Uptake $_{2}$ than it was as an inhibitor of Uptake $_{1}$ (Table 3). Metanephrine was slightly more active than normetanephrine and is the most potent inhibitor of Uptake ${ }_{2}$ so far studied.

The results obtained with hearts from rats treated with antiserum against the nerve growth factor suggest that both uptake processes are associated with sympathetic nerve fibres in the heart. Uptake ${ }_{1}$ was almost completely abolished by this procedure, confirming previous evidence that this process is confined to postganglionic sympathetic nerves (Hertting, Axelrod, Kopin \& Whitby, 1961 ; Hertting \& Schiefthaler, 1964; Marks, Samorajski \& Webster, 1962; Wolfe, Potter, Richardson \& Axelrod, 1962). The accumulation by Uptake $_{2}$ was also greatly reduced, suggesting that this uptake is also associated with postganglionic sympathetic fibres. The residual uptake may be due to a residual uptake capacity of the atrophic sympathetic fibres or possibly to an extraneuronal site of uptake. However, using a fluorescence histochemical technique, Hamberger, Malmfors, Norberg \& Sachs (1964) found no evidence of an extraneuronal uptake of noradrenaline in various tissues of the rat after the administration of noradrenaline to normal animals in vivo. Since large doses of noradrenaline $(0.5$ to $10.0 \mathrm{mg} / \mathrm{kg})$ were used in these experiments, the conditions would have favoured the operation of $\mathrm{Uptake}_{2}$, and the results thus favour the view that Uptake $_{2}$ is confined to sympathetic neurones.

Another feature of Uptake ${ }_{2}$ was the short time within which the uptake was completed As was seen in Fig. 8, the rates of adrenaline and noradrenaline uptake fell off exponentially with time. At a perfusion concentration of $5 \mu \mathrm{g} / \mathrm{ml}$., the half-time of adrenaline uptake was approximately $3 \mathrm{~min}$, and the analysis of adrenaline uptake curves at other perfusion concentrations revealed that in these, too, the half-times were of the order of 1 to $3 \mathrm{~min}$. The disappearance curve for adrenaline shown in Fig. 7 had practically the same half-time 
as the uptake curve. The uptake and disappearance of noradrenaline was also rapid, though there was some indication that the rates were somewhat slower than for adrenaline.

The disappearance of adrenaline and noradrenaline accumulated by Uptake ${ }_{2}$ during perfusion with a catechol amine-free medium could be due to an efflux of the accumulated materials (wash-out) or to a removal by intracellular enzymes. Preliminary experiments in which the catechol amine content of the perfusate outflow was measured during the disappearance of accumulated adrenaline or noradrenaline indicated that the greater part left the heart as unchanged adrenaline or noradrenaline, and the process thus appears to be one of wash-out. The identity of the uptake and wash-out rate constants suggests that the influx of catechol amine occurs at a constant rate and is opposed by an efflux occurring at a rate proportional to the amount of catechol amine accumulated. These conditions would be fulfilled if, for example, an inward transport of catechol amine was opposed by a passive diffusional leakage of the accumulated catechol amine out of the tissue. However, the wash-out of noradrenaline could be completely inhibited by metanephrine, indicating that the efflux is not by diffusional leakage but presumably is also mediated by a transport mechanism. The action of metanephrine in inhibiting wash-out was also interesting since this compound was found to be a potent inhibitor of catechol amine uptake. On the other hand, (-)-metaraminol, which was not an inhibitor of noradrenaline uptake, was equally ineffective as an inhibitor of wash-out. These findings suggest that some common mechanism may underlie both the uptake and wash-out processes. However, the wash-out cannot be mediated by a mechanism exactly similar to that responsible for uptake, since although the uptake was saturable the wash-out showed no indication of having this property.

The wash-out curves for adrenaline and noradrenaline (Fig. 7) show that approximately $2 \mu \mathrm{g}$ of the accumulated catechol amine per $\mathrm{g}$ of tissue remained in the heart even after prolonged periods of wash-out. One possible explanation is that this residual amount represents catechol amines reaccumulated by Uptake $_{1}$ during the wash-out perfusion. This possibility was ruled out by the finding that the addition of (-)-metaraminol to the wash-out medium had no effect on this residual amount of accumulated catechol amine. It has previously been shown that the concentration of metaraminol used was sufficient to inhibit Uptake ${ }_{1}$ completely (Iversen, 1964). It seems probable, therefore, that some of the accumulated catechol amines enter a stable pool within the tissue. However, the rate analysis curves (Figs. $3, b, 8$ ) suggested that, in contrast to what was found with Uptake $e_{1}$, the catechol amines accumulated by Uptake $_{2}$ entered into only one intracellular pool in the tissue. The finding that $\left[{ }^{3} \mathrm{H}\right]$-noradrenaline and net noradrenaline uptakes were virtually identical further suggested that during the uptake of catechol amine there was little or no exchange of the accumulated material with the endogenous noradrenaline content of the tissue. However, a minor accumulation into a second intracellular pool or a partial exchange with endogenous noradrenaline would have been very difficult to detect in the presence of the large amount of catechol amine accumulated in the tissue.

The relation of Uptake, and Uptake Un $_{2}$ to one another remains puzzling. The abrupt emergence of Uptake ${ }_{2}$ by what appears to be a trigger mechanism is illustrated in Figs. 1, 4 and 11. There appears to be virtually no overlap in the operation of the two processes. Thus the kinetic analysis of Uptake ${ }_{1}$ remains valid, since there was no indication that Uptake $_{2}$ was active at low perfusion concentrations. The finding that $(-)$-metaraminol, which was a potent inhibitor of $\mathrm{Uptake}_{1}$, had no effect on the uptake of noradrenaline at 
high perfusion concentrations further suggests that Uptake ${ }_{1}$ is inactive once Uptake ${ }_{2}$ has been activated. The two processes may thus prove to be mutually exclusive in their operation. The activation of Uptake ${ }_{2}$ depended on the extracellular concentration of adrenaline or noradrenaline; lower concentrations of adrenaline than noradrenaline were required in order to produce this effect. It seems likely that the triggering of Uptake ${ }_{2}$ by catechol amines represents a novel pharmacological action of these compounds. This action may also be shared by other related substances and this possibility will be the subject of further investigations.

The discovery of a second uptake process of major quantitative importance was unexpected. The concentrations of catechol amines required to activate Uptake ${ }_{2}$ are sufficiently high to make it appear unlikely that these could be encountered in vivo. However, in several studies of catechol amine uptake in which large doses of catechol amines have been injected in vivo, the resulting extracellular concentrations of adrenaline and noradrenaline would have been sufficiently high to activate Uptake . $_{2}$.

In the original experiments on the uptake of adrenaline and noradrenaline by dog heart in vivo by Raab \& Gigee $(1953,1955)$, massive doses of injected adrenaline and noradrenaline (of the order of $10 \mathrm{mg} / \mathrm{kg}$ ) were used. These doses almost certainly produced plasma concentrations of adrenaline and noradrenaline sufficiently high to have activated Uptake $_{2}$. This conclusion is further supported by the results reported by Raab \& Gigee. They studied the uptake of adrenaline and noradrenaline in heart tissue at short time intervals after injection, and found large uptakes of both amines of the order of $3 \mu \mathrm{g} / \mathrm{g}$ adrenaline and $2 \mu \mathrm{g} / \mathrm{g}$ noradrenaline. It is interesting that the uptake of adrenaline was found to exceed that of noradrenaline and that, in experiments in which the hearts were flushed with a catechol amine-free medium before analysis, a considerable wash-out of adrenaline and a smaller wash-out of noradrenaline was observed. These findings are thus compatible with the properties of Uptake ${ }_{2}$ as described above.

Certain experiments clearly fall into the Uptake ${ }_{2}$ category. Wegmann \& Kako (1961) administered massive doses of noradrenaline to puppies. The accumulated noradrenaline in the heart was analysed $20 \mathrm{~min}$ after the intraperitoneal injection of $10 \mathrm{mg} / \mathrm{kg}$ noradrenaline. An uptake of $5 \mu \mathrm{g}$ noradrenaline per $\mathrm{g}$ of heart was found. These experiments showed that the accumulated noradrenaline was largely located in the cell sap fraction of heart homogenates after differential centrifugation, but the interpretation of these results remains obscure because of the probable involvement of the second catechol amine uptake process. The presence of two catechol amine uptake processes also means that several other studies of catechol amine uptake may need careful re-examination, particularly where large doses of adrenaline or noradrenaline have been used.

In studies of catechol amine uptake it would seem desirable that the doses used should be carefully selected to avoid the possibility of confusion between the two uptake processes. Because of the high catechol amine concentrations required for the operation of Uptake ${ }_{2}$, this process can only be studied satisfactorily in isolated tissue preparations. However,

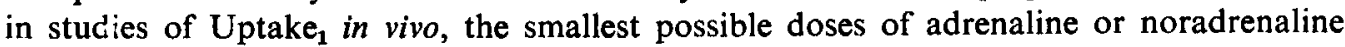
which lead to detectable increases in the tissue content of adrenaline or noradrenaline should be used. Muscholl (1960) has shown that such doses do not have to be very large; he was able to detect a considerable uptake of noradrenaline into various tissues of the rat after the intravenous administration of only 25 to $100 \mu \mathrm{g} / \mathrm{kg}$ noradrenaline. 
Although high concentrations of adrenaline or noradrenaline are required to activate Uptake $_{2}$, it is, nevertheless, possible that this process may have some physiological role. The concentration of noradrenaline in adrenergic nerve endings is very high, and it is possible that extremely high extracellular concentrations of noradrenaline may arise after the release of this noradrenaline into the exceedingly small volume of the synaptic cleft, whose width is from 200 to $500 \AA$ (Eccles, 1964). It is therefore possible that Uptake could serve as an inactivation mechanism for noradrenaline after its release from adrenergic nerve endings. The potentiation of the pharmacological effects of noradrenaline by normetanephrine and metanephrine described by Bacq \& Renson (1961) would support this view, since these compounds were found to act as potent inhibitors of Uptake ${ }_{2}$. It was also found that metanephrine was more potent than normetanephrine in this respect, and this also agrees with Bacq \& Renson's finding that metanephrine was more active than normetanephrine in potentiating the actions of noradrenaline.

Some of the features of the two catechol amine uptake processes described in the rat heart correspond closely with the properties of the catechol amine uptake processes recently described in isolated adrenergic nerve granules by Euler \& Lishajko (1964). These authors found that, at concentrations of less than $1 \mu \mathrm{g} / \mathrm{ml}$. noradrenaline, the uptake of noradrenaline and adrenaline into isolated nerve particles was dependent on the presence of adenosine triphosphate and was stereochemically favourable for the laevoisomers. At higher concentrations the uptake continued by a process which did not require the presence of adenosine triphosphate, which was not stereochemically specific, and which favoured adrenaline rather than noradrenaline. The similarity between these results and the present results is striking but the implications of this correlation are not yet clear.

The present results, which have demonstrated the presence of a second catechol amine uptake process in the rat heart, also correlate well with findings in the mouse in vivo (Iversen $\&$ Whitby, 1962) where a further uptake of $\left[{ }^{3} \mathrm{H}\right]$-noradrenaline and $\left[{ }^{3} \mathrm{H}\right]$-adrenaline was found after the administration of doses larger than those required to saturate a primary uptake process.

\section{SUMMARY}

1. The uptake of noradrenaline and adrenaline by the isolated heart of the rat was studied after perfusion with a medium containing high concentrations of ( \pm )-adrenaline or ( \pm -noradrenaline ( 1 to $40 \mu \mathrm{g} / \mathrm{ml}$.). Under these conditions a novel catechol amine uptake process (Uptake ${ }_{2}$ ) was activated which had different properties from the uptake process previously described in this preparation (Uptake $)_{1}$ which operated at lower perfusion concentrations of adrenaline or noradrenaline.

2. The initial rates of catechol amine uptake during perfusion with various concentrations of adrenaline or noradrenaline satisfied Michaelis-Menten kinetics with a $K_{m}$ for ( \pm )-noradrenaline of $252.0 \times 10^{-6} \mathrm{M}\left(42.6 \mu \mathrm{g} / \mathrm{ml}\right.$.) and for $( \pm)$-adrenaline of $51.6 \times 10^{-6} \mathrm{M}(9.5$ $\mu \mathrm{g} / \mathrm{ml}$.). In contrast to Uptake $_{1}, \mathrm{Uptake}_{2}$ favoured the accumulation of adrenaline rather than noradrenaline, and exhibited no stereochemical specificity for the ( + )- and (-)stereoisomers of adrenaline or noradrenaline.

3. There were striking differences in the drug sensitivities of the two uptake processes. (-)-Metaraminol, which was a potent inhibitor of noradrenaline accumulation by Uptake, had no effect on Uptake $_{2}$. On the other hand, $( \pm)$-normetanephrine and $( \pm)$-metanephrine 
were potent inhibitors of noradrenaline accumulation by Uptake ${ }_{2}$, though these compounds were only weakly active as inhibitors of Uptake . $_{\text {. }}$

4. A large part of the catechol amines accumulated by Uptake $_{2}$ was rapidly washed out of the tissue if perfusion was continued with a catechol amine-free medium, but a residue of approximately $2 \mu \mathrm{g}$ catechol amine per $\mathrm{g}$ of tissue remained in the heart even after prolonged periods of wash-out. The wash-out of noradrenaline was completely suppressed by $( \pm)$-metanephrine, indicating that the wash-out process was not a passive diffusional leakage.

5. Uptake ${ }_{2}$ was entirely inactive at low external catechol amine concentrations and appeared to be triggered into operation at certain critical concentrations of adrenaline or noradrenaline. The results of preliminary experiments with hearts from "immunosympathectomized" rats indicated that both uptake processes are associated with sympathetic nerve fibres in the heart.

I am grateful to the Medical Research Council for a scholarship and for a grant for animals and materials, and to the following for their generous gifts of materials: Dr E. Muscholl, University of Mainz, $(+)-$ adrenaline; Dr J. Raventos, I.C.I., (+)-noradrenaline and phenoxybenzamine; Geigy Pharmaceuticals, desipramine; and Merck, Sharpe \& Dohme, (-)-metaraminol. I also wish to thank Professor A. S. V. Burgen for much helpful advice and criticism, and Professor E. Zaimis for preparing the "immunosympathectomized " animals and placing them at my disposal.

\section{REFERENCES}

BACQ, Z. M. \& RENSON, J. (1961). Actions et importance physiologique de la métanéphrine et de la normétanéphrine. Arch. int. Pharmacodyn., 130, 385-402.

ECCLES, J. C. (1964). The Physiology of Synapses, p. 23. Berlin: Springer-Verlag.

EULER, U. S. VON \& LISHAJKO, F. (1964). Uptake of L- and D-isomers of catecholamines in adrenergic nerve granules. Acta physiol. scand., 60, 217-222.

HAMBERGER, B., MALMFORS, T., NORBERG, K-A. \& SACHS, C. (1964). Uptake and accumulation of catecholamines in peripheral adrenergic neurons of reserpinized animals, studies with a histochemical method. Biochem. Pharmacol., 13, 841-844.

HERTTING, G., AXELROD, J., KOPIN, I. J. \& WhITBY, L. G. (1961). Lack of uptake of catecholamines after chronic denervation of sympathetic nerves. Nature (Lond.), $189,66$.

HERTTING, G. \& SCHIEFTHALRR, T. (1964). The effect of stellate ganglion excision on the catecholamine content and the uptake of $\mathrm{H}^{3}$-norepinephrine in the heart of the cat. Int. J. Neuropharmacol., 3, 65-69.

IVERSEN, L. L. (1963). The uptake of noradrenaline by the isolated perfused rat heart. Brit. J. Pharmacol., 21, 523-537.

IVERSEN, L. L. (1964). Inhibition of noradrenaline uptake by sympathomimetic amines. J. Pharm. Pharmacol., 16, 435-439.

IVERSEN, L. L. (1965). The uptake of adrenaline by the isolated rat heart. Brit.J. Pharmacol., 24, 387-394.

IVERSEN, L. L. \& WHITBY, L. G. (1962). Retention of injected catechol amines by the mouse. Brit. J. Pharmacol., 19, 355-364.

LEVI-MONTALCINI, R. \& ANGeletti, P. U. (1962). Noradrenaline and monoaminoxidase content in immunosympathectomized animals. Int. J. Neuropharmacol., 1, 161-164.

MARKS, B. H., SAMORAJSKI, T. \& WEBSTER, E. J. (1962). Radioautographic localization of norepinephrine- $\mathrm{H}^{3}$ in the tissues of mice. J. Pharmacol. exp. Ther., 138, 376-381.

Morgan, H. E., Henderson, M. J., RegeN, D. M. \& PARK, C. R.'(1961). Regulation of glucose uptake in muscle. I. The effects of insulin and anoxia on glucose transport and phosphorylation in the isolated perfused heart of normal rats. J. biol. Chem., 236, 253-261.

MusCHOLL, E. (1960). Die Hemmung der Noradrenaline-Aufnahme des Herzens durch Reserpin und die Wirkung von Tyramin. Naunyn-Schmiedeberg's Arch. exp. Path. Pharmak., 240, 234-241.

PeARL, R. (1940). Medical Biometry and Statistics, p. 450. Philadelphia: Saunders.

RAAB, W. \& GIGEE, W. (1953). Die Katecholamine des Herzens. Naunyn-Schmiedeberg's Arch. exp. Path. Pharmak., 219, 248-262.

RAAB, W. \& GIGEE, W. (1955). Specific avidity of the heart muscle to absorb and store epinephrine and norepinephrine. Circulat. Res., 3, 553-558.

WeOMANN, A. \& KAKo, K. (1961). Particle-bound and free catecholamines in dog hearts and the uptake of injected norepinephrine. Nature (Lond.), 192, 978.

Wolfe, D. E., PotTer, L. T., Richardson, K. C. \& AXelrod, J. (1962). Localizing tritiated norepinephrine in sympathetic axons by electron microscopic autoradiography. Science, 138, 440-441. 